\title{
Neovascular PSMA expression is a common feature in malignant neoplasms of the thyroid
}

\author{
Birthe Heitkötter ${ }^{1, *}, K$ Konrad Steinestel ${ }^{2, *}$, Marcel Trautmann $^{1}$, Inga Grünewald ${ }^{1}$, \\ Peter Barth ${ }^{1}$, Heidrun Gevensleben ${ }^{3}$, Martin Bögemann ${ }^{4}$, Eva Wardelmann ${ }^{1}$, \\ Wolfgang Hartmann ${ }^{1}$, Kambiz Rahbar ${ }^{5}$ and Sebastian Huss ${ }^{1}$ \\ ${ }^{1}$ Gerhard Domagk Institute of Pathology, University of Münster, Münster, Germany \\ ${ }^{2}$ Institute of Pathology, Military Hospital UIm, Ulm, Germany \\ ${ }^{3}$ Institute of Pathology, Neuss, Germany \\ ${ }^{4}$ Department of Urology, University Hospital Münster, University of Münster, Münster, Germany \\ ${ }^{5}$ Department of Nuclear Medicine, University Hospital Münster, Münster, Germany \\ *These authors contributed equally to this work
}

Correspondence to: Sebastian Huss, email: sebastian.huss@ukmuenster.de

Keywords: PSMA; thyroid cancer; tumor neoangiogenesis; prostate cancer

Received: July 26, 2017 Accepted: November 15, $2017 \quad$ Published: January 04, 2018

Copyright: Heitkötter et al. This is an open-access article distributed under the terms of the Creative Commons Attribution License 3.0 (CC BY 3.0), which permits unrestricted use, distribution, and reproduction in any medium, provided the original author and source are credited.

\section{ABSTRACT}

Aim: PSMA (prostate-specific membrane antigen) is physiologically expressed in normal prostate tissue and over expressed in prostate cancer cells, therefore constituting a potential target for antibody-based radioligand therapy. Very recent imaging findings reported PSMA-PET/CT uptake in various thyroid lesions. We were therefore encouraged to systematically analyse PSMA expression in different benign and malignant thyroid lesions.

Methods: Immunohistochemistry was used to detect PSMA expression in 101 thyroid lesions, while neovasculature was identified by CD34 immunostaining.

Results: PSMA expression in the neovasculature was significantly more frequent in malignant tumors $(36 / 63 ; 57.1 \%)$ compared to benign diseases $(5 / 38 ; 13.2 \%$; $p$ $=0.0001)$. In addition, PSMA expression levels in the neovasculature of poorly and undifferentiated thyroid cancers were significantly higher compared to differentiated thyroid tumors $(p=0.021)$. However, one case with a strong expression in follicular adenoma was identified.

Conclusions: We conclude that neovascular PSMA expression is common in thyroid cancer but may also rarely be found in benign thyroid diseases, such as follicular adenoma. High expression in the tumor-associated neovasculature is predominantly found in poorly differentiated and undifferentiated (anaplastic) thyroid cancer. This knowledge is highly relevant when interpreting PSMA/PET-CT scans from patients with prostate cancer. In addition, our findings might provide a rationale for further evaluation of PSMA-targeted anti-neovascular or radioligand therapy in metastatic dedifferentiated thyroid cancer.

\section{INTRODUCTION}

PSMA (prostate specific membrane antigen) is a $100 \mathrm{kDa}$ type II-transmembrane glycoprotein with both intra- and extracellular protein domains exerting folate hydrolase and neurocarboxypeptidase activity $[1,2]$. It was originally reported to be physiologically expressed by prostate cells [3, 4].A Strong upregulation in prostate cancer cells, which was further described, led to the development of PSMA-based imaging technologies for the detection of metastatic disease in advanced prostate cancer. PSMA-based radioligand therapy has furthermore been established as a therapeutic regimen in metastatic prostate cancer [2, 5-11]. In addition, PSMA was found 
to be expressed in the endothelium of tumor-associated neovasculature in some solid malignancies (breast, lung and urothelial cancer), possibly due to the effect of tumorassociated angiogenic factors [12-16].

Functional studies revealed that the role of PSMA in tumor angiogenesis is part of an autoregulatory loop involving $\beta 1$-integrin and p21-activated kinase 1 (PAK1). In this context, active PSMA facilitates endothelial cell invasion through the extracellular matrix by interacting with the cytoskeleton via integrin signaling and actinbinding protein Filamin A [7, 17].

Very recently, PSMA expression has also been reported in thyroid cancer [18-20]. The authors of these studies described the detection of differentiated follicular and papillary thyroid cancer using PSMA-PET/CT Imaging. As thyroid cancer has not been systematically analysed for PSMA expression, the current study aims at investigating different entities of thyroid malignancies, including papillary, follicular, medullary and undifferentiated (anaplastic) thyroid carcinomas. We also included benign and inflammatory lesions, such as adenomas and thyroiditis as well as sporadic nodular goiter, since Gordon et al. recently reported increased PSMA expression in nonneoplastic, regenerative and reparative neovasculature [7].

\section{RESULTS}

\section{PSMA expression in benign thyroid diseases}

In a patient with biopsy-confirmed acinar adenocarcinoma of the prostate (Gleason score: $4+5=9$; grade group 5 (ISUP)) ${ }^{68} \mathrm{Ga}$-PSMA PET/CT revealed bone metastases, nodal involvement and a nodule in the left thyroid lobe showing a focal uptake. The thyroid lesion was regarded as being suspicious for thyroid cancer, as metastases to the thyroid are extremely uncommon in prostate cancer patients. To rule out a second malignancy, hemithyroidectomy was performed. However, the nodule was classified as benign thyroid adenoma. A strong (neo-) vascular PSMA expression was noted, providing a good explanation for the observed ${ }^{68} \mathrm{Ga}$-PSMA PET/CT uptake.

In our immunohistochemical assessment, PSMA expression in the neovasculature was found to be positive in $5 / 38$ benign thyroid diseases $(13.2 \%)$ : 0/18 Sporadic nodular goiter, 2/9 follicular adenoma, 1/2 hyalinising trabecular thyroid tumor, 1/3 Grave's Disease, 1/2 Lymphocytic thyroiditis, 0/1 Granulomatous thyroiditis, $0 / 3$ unspecific thyroiditis. The majority of these cases $(4 / 38,10.5 \%)$ presented with low expression levels, while the patient diagnosed with follicular adenoma showed a strong PSMA expression (Table 1 and Figures 1 and 2).

\section{PSMA expression in thyroid cancer}

PSMA expression of the tumor-associated neovasculature was found in 36/63 malignant thyroid tumors (57.1\%): 18/31 papillary thyroid carcinoma, 4/10 follicular carcinoma, 4/12 medullary carcinoma, 6/6 poorly-differentiated thyroid carcinoma and 4/4 anaplastic thyroid carcinoma. In these malignant tumors, low expression levels could be observed in 9/31 papillary, $2 / 10$ follicular, 4/12 medullary and 2/6 poorly-differentiated thyroid carcinomas $(17 / 63 ; 37.0 \%)$, whereas strong PSMA expression of the neovasculature was noted in nine papillary, two follicular, four poorly-differentiated and four anaplastic carcinomas $(19 / 63 ; 30.2 \%$ ) (Table 1 and Figure 2).

We then performed a histogram analysis linking the biological potential of the different thyroid diseases to the neovascular PSMA labelling index (Figure 3). The overall PSMA expression (low and high expression, labelling index 1 and 2) was found in the neovasculature of 36/63 (57.1\%) malignant tumors, whereas overall PSMA expression in the vasculature of benign diseases $(5 / 38 ; 13.2 \%)$ was significantly lower $(p=0.0001$, Fisher's exact test $)$.

Analysing only diseases with strong neovascular PSMA expression (labelling index 2), malignant tumors presenting with a high PSMA expression were more frequent $(19 / 63 ; 30.2 \%)$ compared to benign diseases $(1 / 38 ; 2.63 \%)(p=0.0006$, Fisher's exact test $)$.

Focusing only on the PSMA expression in the neovasculature of malignant thyroid diseases, we noted that the neovasculature of undifferentiated thyroid cancers (poorly-differentiated and anaplastic carcinoma) showed strong PSMA expression in 8/10 (80.00\%) cases, while high neovascular PSMA was only rarely observed in differentiated carcinomas (papillary, follicular and medullary thyroid cancers) (11/53 or $20.8 \%$ of cases; $p$ $=0.0006$, Fisher's exact test). Detailed data on staining intensity and histological distribution patterns for each evaluated specimen is shown in Supplementary Table 1.

\section{DISCUSSION}

Apart from its known strong expression in prostatic epithelium and prostate cancer cells, PSMA is expressed in the tumor neovasculature of different tumor types, including renal cell carcinomas, bladder carcinoma, colonic adenocarcinomas, glioblastoma multiforme, lung cancers, malignant melanomas and soft tissue tumors [12-14, 21-23].

Very recently, PSMA uptake in patients undergoing PSMA PET/CT has been reported in cases of thyroid cancer, such as papillary and follicular carcinoma [18, 19]. In our present study, we report on a patient with metastatic prostate cancer, where ${ }^{68} \mathrm{Ga}-\mathrm{PSMA}$ PET/ $\mathrm{CT}$ revealed a nodule in the left thyroid lobe with a focal uptake being suspicious for thyroid cancer. After hemithyroidectomy and histopathological workup, the nodule was classified as benign thyroid adenoma. A strong (neo-) vascular PSMA expression was noted, providing a good explanation for the observed ${ }^{68} \mathrm{Ga}-$ 
Table 1: Cases with strong PSMA expression in the (neo-) vasculature (PSMA labelling index = 2; total $n=19$ )

\begin{tabular}{lll}
\hline Biological Potential & $\boldsymbol{n}$ & Cases \\
\hline Malignant & 9/31 Papillary thyroid carcinoma \\
& 2/10 Follicular thyroid carcinoma \\
& $19 \quad$ 0/12 Medullary thyroid carcinoma \\
& 4/6 Poorly differentiated thyroid carcinoma \\
& 4/4 Dedifferentiated (anaplastic) thyroid carcinoma \\
& 0/18 Sporadic nodular goiter \\
& 1/9 Follicular Adenoma \\
& 0/2 Hyalinising trabecular thyroid tumor \\
& 0/3 Grave's Disease \\
& 0/2 Lymphocytic thyroiditis \\
& 0/1 Granulomatous thyroiditis \\
& 0/3 Unspecific thyroiditis \\
\hline
\end{tabular}

PSMA PET/CT uptake. This finding prompted us to systematically analyse different thyroid carcinomas for neovascular as well as intratumoral PSMA expression. We included samples of benign thyroid disease, such as sporadic nodular goiter, follicular adenoma and samples of thyroiditis. We expected to find PSMA expression here as well, as Gordon et al. reported PSMA expression in nonneoplastic, regenerative, and reparative neovasculature [7]. Indeed, we found PSMA expression in a subset of benign thyroid diseases. However, PSMA expression was observed significantly more often in the neovasculature of malignant thyroid tumors $(36 / 63 ; 57.1 \%)$ compared to the vasculature of benign diseases $(5 / 38 ; 13.2 \% ; p=0.0001)$. As expected, our initial patient with follicular adenoma of

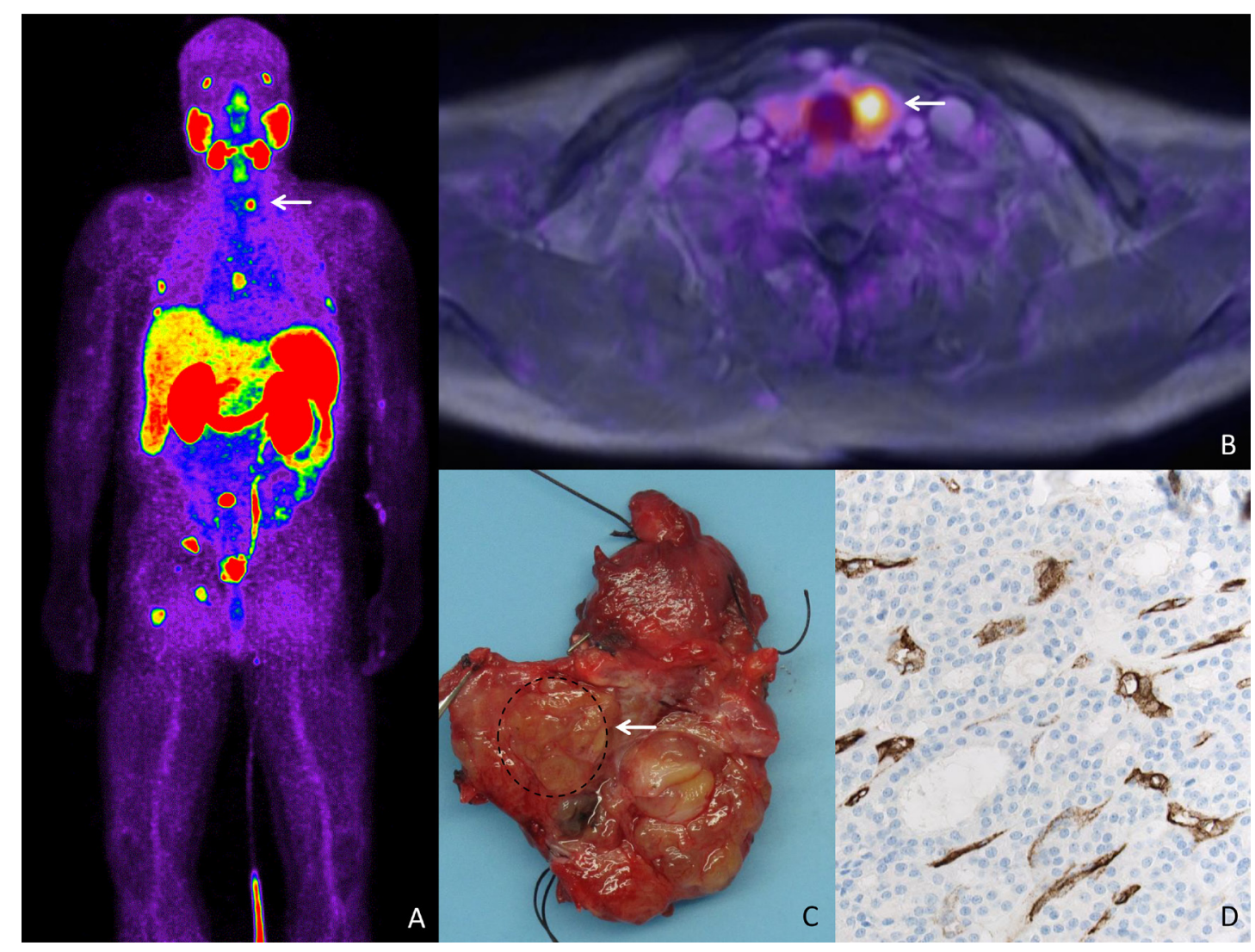

Figure 1: PSMA expression in a patient with a metastatic prostate cancer and thyroid adenoma. (A) Bodyscan of PSMAPet/CT revealing uptake in several bone lesions in a patient with metastatic prostate cancer. Focal uptake is seen in the left thyroid lobe (arrow). (B) Transaxial image of fused 68 Ga-PSMA-PET/MRI showing focal uptake in a nodule in the left thyroid lobe. (C) Gross section of the thyroid lobe revealing a nodule that was histologically classified as adenoma. (D) Strong PSMA expression (200x, IHC) is shown in the adenoma-associated vasculature, apparently being responsible for the PSMA-PET/MRI uptake. 
the thyroid revealed high PSMA expression that was also visible on PSMA PET/CT scan. A similar case has also recently been reported in the literature by others [24]. Very recently, Bychkov et al. investigated PSMA expression in thyroid tumors and reported comparable results. The authors describe neovascular PSMA expression in more than $50 \%$ of their thyroid cancer cases. They furthermore recognized microvascular PSMA expression in $19 \%$ of follicular adenomas [25].

In our cohort, PSMA expression in the neovasculature of poorly and undifferentiated thyroid cancer was shown to be significantly higher than in differentiated thyroid cancer $(p=0.021)$. The biological significance of this finding is unclear but might be due to intratumoral hypoxia as a result of rapid growth of these neoplasms. Since PSMA facilitates endothelial cell invasion during angiogenic sprouting, PSMA upregulation may enhance tumor vascularization, supporting tumor growth by provision of oxygen and nutrients [7, 17]. Therefore, targeting PSMA-expressing neovessels might represent a promising therapeutic option in rapidly growing solid tumors. In our study we used an anti-PSMA antibody (subclone 3E6) targeting the extracellular region of PSMA. In contrast to other subclones, this might be

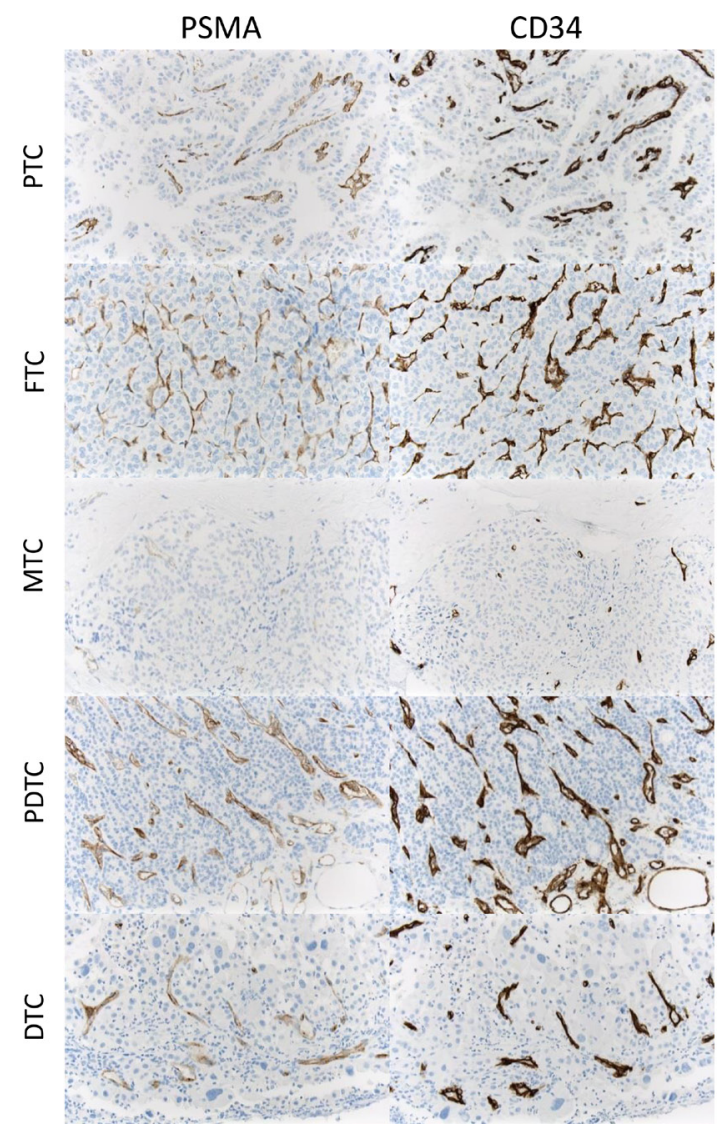

better used to predict the likelihood of success of PSMA imaging and therapy. For patients with metastatic prostate cancer, PSMA-targeted radionuclide therapy has been established as a therapeutic and diagnostic option [5, 26]. PSMA-617, which was developed by the German Cancer Research Centre (DKFZ) in Heidelberg, seems to be a promising ligand for diagnostics and therapy of prostate cancer metastases and recurrences. Different studies using Lutetium-177 labeled PSMA-617 in patients with metastatic prostate cancer have shown convincing response rates and acceptable toxicity profiles [6, 27]. Future studies therefore need to investigate possible antiangiogenic effects of PSMA-targeted radionuclide therapy in tumors with PSMA-expressing neovessels especially in metastatic dedifferentiated thyroid cancer, where radioactive iodine therapy becomes ineffective to control disease. Two recent studies have evaluated PSMA-targeted therapies in different solid cancer subtypes. Milowsky et al. recently showed that tumor-associated neovasculature in multiple advanced metastatic solid cancers could be targeted with an Indium-111- labeled PSMA antibody [28]. Another phase I study using the PSMA-targeted docetaxel-containing nanoparticle BIND-014 in patients with advanced solid tumors was recently performed [29].

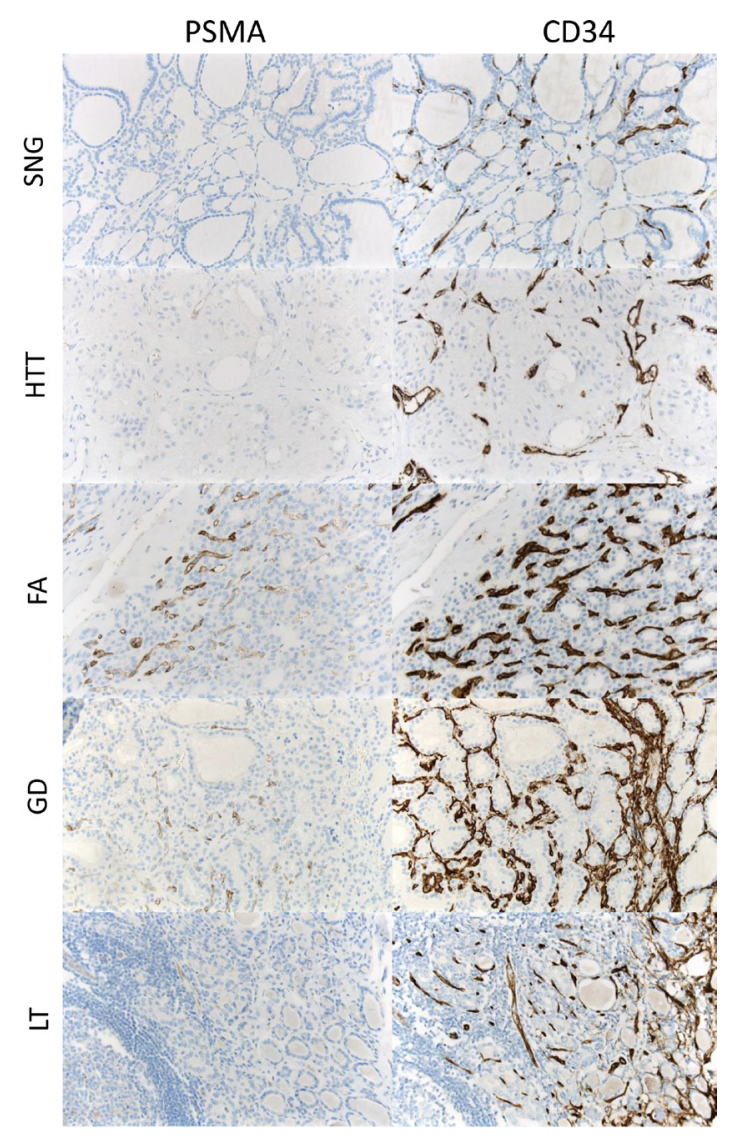

Figure 2: PSMA expression in malignant tumors and benign thyroid diseases. (Neo-)vascular PSMA expression in different thyroid cancer subtypes and benign thyroid disease (PTC, papillary thyroid cancer; FTC: follicular thyroid cancer; MTC, medullary thyroid cancer; PDTC, poorly differentiated thyroid cancer; DTC, dedifferentiated (anaplastic) thyroid cancer; SNG, sporadic nodular goiter; FA, follicular adenoma; HTT, hyalinising trabecular thyroid tumor; GD, Grave's disease; LT, lymphocytic thyroiditis). Vasculature was identified by means of CD31 and CD34 coexpression. 
Our finding of neovascular PSMA expression in thyroid carcinomas might point towards a potential therapeutic use of PSMA-targeted radioligands or antibody-based antiangiogenic agents, especially when tumors dedifferentiate and iodine-based radiotherapy becomes ineffective to control disease. In line with these results, strong PSMA expression was noted in poorly and undifferentiated thyroid carcinomas. However, future studies have to evaluate internalization and retention of radiolabeled PSMA ligands in metastatic dedifferentiated thyroid cancer for a possible radioligand therapy in these patients. In addition, our study reveals that neovascular PSMA expression can be observed in both malignant and benign thyroid diseases. This knowledge is important for pathologists, clinicians and radiologists when interpreting PSMA/PET-CT scans from patients with prostate cancer, as this finding is apparently responsible for tracer uptake that must not been misinterpreted as prostate cancer metastasis.

\section{MATERIALS AND METHODS}

\section{Cases}

A total of 101 cases with different thyroid diseases were selected by two experienced pathologists (Table 2), and whole sections (one per case) from paraffin-embedded formalinfixed tissues were stained with PSMA, CD34 and CD31. One patient with biopsy-confirmed prostate cancer was included. ${ }^{68} \mathrm{Ga}$-PSMA-PET/CT was performed to rule out metastases. The indication for ${ }^{68} \mathrm{Ga}$-PSMA-imaging was appointed by an interdisciplinary tumor board, and the patient gave informed consent to the use of his data in this study. The study was approved by the local ethics committee (Az. 2016-445-f-S).

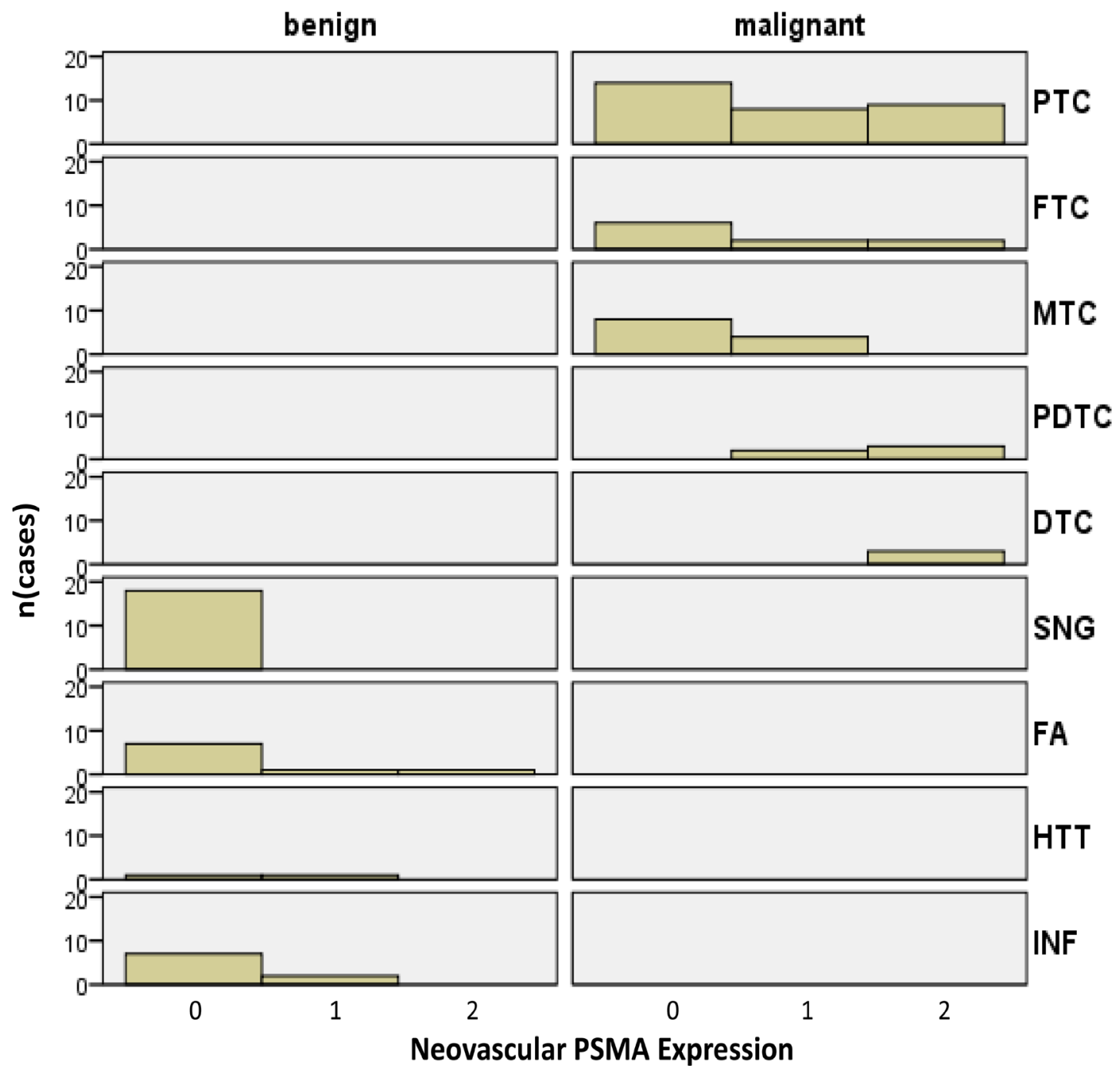

Figure 3: Histograms of malignant tumors and benign thyroid diseases according to their biological potential and PSMA labelling index. Histograms of malignant tumors and benign thyroid diseases according to their biological potential and PSMA labelling index (PTC, papillary thyroid cancer; FTC: follicular thyroid cancer; MTC, medullary thyroid cancer; PDTC, poorly differentiated thyroid cancer; DTC, dedifferentiated (anaplastic) thyroid cancer; SNG, sporadic nodular goiter; FA, follicular adenoma; HTT, hyalinising trabecular thyroid tumor; INF, inflammatory diseases). 
Table 2: Malignant neoplasms and benign diseases included in the present study $(n=101)$

\begin{tabular}{ll}
\hline Biological Potential & Cases \\
\hline Malignant & Papillary thyroid carcinoma (31) \\
& Follicular thyroid carcinoma (10) \\
& Medullary thyroid carcinoma (12) \\
& Poorly differentiated thyroid carcinoma (6) \\
& Dedifferentiated (anaplastic) thyroid carcinoma (4) \\
& Sporadic nodular goiter (18) \\
Benign & Follicular Adenoma (9) \\
& Hyalinising trabecular thyroid tumor (2) \\
& Grave's Disease (3) \\
& Lymphocytic thyroiditis (2) \\
& Granulomatous thyroiditis (1) \\
& Unspecific thyroiditis (3) \\
\hline
\end{tabular}

\section{Immunohistochemistry}

Immunhistochemistry (IHC) was performed on $4-\mu \mathrm{m}$-thick paraffin sections using the peroxidaseconjugated avidin-biotin method. Antibodies included a monoclonal mouse anti-PSMA antibody (clone 3E6, Ventana, Germany, 1:50 dilution), monoclonal anti-CD34 antibody (clone QBEnd10, Ventana, Germany, ready to use concentration of $0.8 \mu \mathrm{g} / \mathrm{ml}$ ) and anti-CD31 antibody (clone JC70, Cell Marque, United States, concentration of $0.61 \mu \mathrm{g} / \mathrm{ml}$ ). Several subclones of anti-PSMA antibodies have been evaluated and commonly used subclones include 7E11 and 3E6. We decided to use clone 3E6 as it targets an extracellular epitope of PSMA. For imaging or therapeutic purposes only antibodies targeting extracellular domains can be potentially used as they have to be internalized by living cells.

In brief, sections were deparaffinized in xylene and rehydrated through graded ethanol at room temperature. Incubation with the primary antibodies was performed for 30 minutes at room temperature. After washing, the sections were incubated with biotinylated secondary antibodies. Immunoreactions were visualized using a 3-amino-9ethylcarbazole as a substrate (Ventana Optiview DAB IHC detection KIT, Ref: 760-700, Germany). Prostate carcinoma tissue sections served as a positive control.

\section{Assessment of PSMA expression}

PSMA expression was evaluated by two experienced pathologists ( $\mathrm{BH}$ and $\mathrm{KS}$ ) on immunostained whole slides. In malignant diseases, tumor cells and associated neovascular endothelium were analysed separately, and the identity of vascular structures was confirmed by CD31 and CD34 coexpression, a common marker for endothelial cells [30-32]. In benign diseases, PSMA expression was analysed in preexistent vascular capillary structures.

In malignant diseases, any reactivity in either tumor cells or tumor-associated vessels was considered positive. Staining intensity was scored semiquantitatively as negative (0), weak ( 1 = barely perceptible staining at high power (400x) magnification), moderate $(2=$ readily apparent at low power (40x) magnification) or strong (3). The fraction of PSMA positive cells was assessed as $<5 \%$ or $>5 \%$. In the case of heterogeneous staining, the predominant pattern was recorded. For further analysis, labelling indices were defined. A weak (1) or moderate (2) staining intensity in $<5 \%$ of the neovasculature and a weak (1) staining intensity in $>5 \%$ of the neovasculature was allocated to the "low expression" group (PSMA labelling index $=1$ ), whereas a moderate (2) staining intensity in $>5 \%$ of the neovasculature and a strong (3) staining intensity in $<$ or $>5 \%$ of the neovasculature were assigned to the "strong expression" group (PSMA labelling index $=2$ ). In benign diseases, PSMA expression was studied in benign tissue and the preexisting vessels using the algorithms described above. This scoring system has been previously established in soft tissue tumors [23, 33].

\section{${ }^{68}$ Ga-PSMA-PET/CT}

Whole-body PET/CT was performed $65.1 \pm 7.0$ min after injection of $161 \pm 19.8 \mathrm{MBq}$ (range, 131-193 $\mathrm{MBq}$ ) of ${ }^{68} \mathrm{Ga}-\mathrm{PSMA}-\mathrm{HBED}-\mathrm{CC}$ (HBED-CC is $N, N^{\prime}$ bis [2-hydroxy-5(carboxyethyl)benzyl] ethylenediamine$N, N^{\prime}$-diacetic acid) [26]. The patient was asked to void immediately before undergoing scanning. The scans were obtained using a high-resolution hybrid PET/CT system (Biograph mCT, with a 128-slice CT component; Siemens Medical Solutions). Low-dose CT of the entire area covered by PET (from skull to mid-thigh) was performed for attenuation correction. After completion of the CT scan, PET data were acquired for $3 \mathrm{~min}$ per bed position. PET images were reconstructed using the standard manufacturer-supplied software (PET resolution of $3 \mathrm{~mm}$ ).

\section{Statistics}

SPSS-21 software (IBM, Armonk, NY, USA) was used. Fisher's exact test was used if appropriate. All tests were two-sided with a $95 \%$ confidence interval. A $p$-value of $\leq 5 \%$ was considered statistically significant. 


\section{Abbreviations}

DTC - dedifferentiated (anaplastic) thyroid cancer; FA - follicular adenoma; FTC - follicular thyroid cancer; GD - Grave's disease; GT - granulomatous thyroiditis; HTT hyalinising trabecular thyroid tumor; LT - lymphocytic thyroiditis; MTC - medullary thyroid cancer; PDTC poorly differentiated thyroid cancer; PSMA - prostate specific membrane antigen; PTC - papillary thyroid cancer; SNG - sporadic nodular goiter; UT - unspecific thyroiditis.

\section{Author contributions}

$\mathrm{BH}, \mathrm{KS}, \mathrm{KR}$, and SH conceived and coordinated the study. BH, KS and SH drafted the manuscript. MT, WH, IG, PB, HG, MB, EW were involved in data analysis and interpretation. All authors reviewed the manuscript.

\section{ACKNOWLEDGMENTS}

We thank Inka Buchroth (Münster) for technical assistance.

\section{CONFLICTS OF INTEREST}

The authors declare that there is no conflicts of interests associated with this manuscript.

\section{GRANT SUPPORT}

The Deutsche Forschungsgemeinschaft (DFG) provided financial support to KS (grant STE 2467/1-1).

\section{REFERENCES}

1. Carter RE, Feldman AR, Coyle JT. Prostate-specific membrane antigen is a hydrolase with substrate and pharmacologic characteristics of a neuropeptidase. Proc Natl Acad Sci U S A. 1996; 93:749-53.

2. Pinto JT, Suffoletto BP, Berzin TM, Qiao CH, Lin S, Tong WP, May F, Mukherjee B, Heston WD. Prostate-specific membrane antigen: a novel folate hydrolase in human prostatic carcinoma cells. Clin Cancer Res. 1996; 2:1445-51.

3. Troyer JK, Beckett ML, Wright GL Jr. Detection and characterization of the prostate-specific membrane antigen (PSMA) in tissue extracts and body fluids. Int J Cancer. $1995 ; 62: 552-8$.

4. Silver DA, Pellicer I, Fair WR, Heston WD, Cordon-Cardo C. Prostate-specific membrane antigen expression in normal and malignant human tissues. Clin Cancer Res. 1997; 3:81-5.

5. Ahmadzadehfar H, Rahbar K, Kurpig S, Bogemann M, Claesener M, Eppard E, Gartner F, Rogenhofer S, Schafers M, Essler M. Early side effects and first results of radioligand therapy with (177)Lu-DKFZ-617 PSMA of castrate-resistant metastatic prostate cancer: a two-centre study. EJNMMI Res. 2015; 5:114. https://doi.org/10.1186/ s13550-015-0114-2.

6. Kratochwil C, Giesel FL, Stefanova M, Benesova M, Bronzel M, Afshar-Oromieh A, Mier W, Eder M, Kopka $\mathrm{K}$, Haberkorn U. PSMA-targeted radionuclide therapy of metastatic castration-resistant prostate cancer with Lu-177 labeled PSMA-617. J Nucl Med. 2016; 57:1170-76. https:// doi.org/10.2967/jnumed.115.171397.

7. Ghosh A, Heston WD. Tumor target prostate specific membrane antigen (PSMA) and its regulation in prostate cancer. J Cell Biochem. 2004; 91:528-39. https://doi.org/ 10.1002/jcb.10661.

8. Bostwick DG, Pacelli A, Blute M, Roche P, Murphy GP. Prostate specific membrane antigen expression in prostatic intraepithelial neoplasia and adenocarcinoma: a study of 184 cases. Cancer. 1998; 82:2256-61.

9. Evangelista L, Briganti A, Fanti S, Joniau S, Reske S, Schiavina R, Stief C, Thalmann GN, Picchio M. New Clinical Indications for F/C-choline, New Tracers for Positron Emission Tomography and a Promising Hybrid Device for Prostate Cancer Staging: A Systematic Review of the Literature. Eur Urol. 2016; 70:161-75. https://doi. org/10.1016/j.eururo.2016.01.029.

10. Foss CA, Mease RC, Cho SY, Kim HJ, Pomper MG. GCPII imaging and cancer. Curr Med Chem. 2012; 19:1346-59.

11. Bander NH, Trabulsi EJ, Kostakoglu L, Yao D, Vallabhajosula S, Smith-Jones P, Joyce MA, Milowsky M, Nanus DM, Goldsmith SJ. Targeting metastatic prostate cancer with radiolabeled monoclonal antibody J591 to the extracellular domain of prostate specific membrane antigen. J Urol. 2003; 170:1717-21. https://doi.org/10.1097/01. ju.0000091655.77601.0c.

12. Chang SS, Reuter VE, Heston WD, Bander NH, Grauer LS, Gaudin PB. Five different anti-prostate-specific membrane antigen (PSMA) antibodies confirm PSMA expression in tumorassociated neovasculature. Cancer Res. 1999; 59:3192-8.

13. Wang HL, Wang SS, Song WH, Pan Y, Yu HP, Si TG, Liu Y, Cui XN, Guo Z. Expression of prostate-specific membrane antigen in lung cancer cells and tumor neovasculature endothelial cells and its clinical significance. PLoS One. 2015; 10:e0125924. https://doi.org/10.1371/journal. pone. 0125924 .

14. Wernicke AG, Edgar MA, Lavi E, Liu H, Salerno P, Bander $\mathrm{NH}$, Gutin PH. Prostate-specific membrane antigen as a potential novel vascular target for treatment of glioblastoma multiforme. Arch Pathol Lab Med. 2011; 135:1486-9. https://doi.org/ 10.5858/arpa.2010-0740-OA.

15. Godeiro KD, Frota AC, Antecka E, Odashiro AN, Maloney $\mathrm{S}$, Fernandes B, Burnier MN Jr. Prostate-specific membrane antigen is undetectable in choroidal neovascular membrane. $\mathrm{J}$ Carcinog. 2006; 5:21. https://doi.org/ 10.1186/1477-3163-5-21.

16. Chang SS, O'Keefe DS, Bacich DJ, Reuter VE, Heston WD, Gaudin PB. Prostate-specific membrane antigen is 
produced in tumor-associated neovasculature. Clin Cancer Res. 1999; 5:2674-81.

17. Conway RE, Petrovic N, Li Z, Heston W, Wu D, Shapiro LH. Prostate-specific membrane antigen regulates angiogenesis by modulating integrin signal transduction. Mol Cell Biol. 2006; 26:5310-24. https://doi.org/10.1128/ MCB.00084-06.

18. Taywade SK, Damle NA, Bal C. PSMA Expression in Papillary Thyroid Carcinoma: Opening a New Horizon in Management of Thyroid Cancer? Clin Nucl Med. 2016; 41:e263-5. https://doi.org/10.1097/ RLU.0000000000001148.

19. Sager S, Vatankulu B, Uslu L, Sonmezoglu K. Incidental Detection of Follicular Thyroid Carcinoma in 68Ga-PSMA PET/CT Imaging. J Nucl Med Technol. 2016; 44:199-200. https://doi.org/10.2967/jnmt.115.171660.

20. Lutje S, Gomez B, Cohnen J, Umutlu L, Gotthardt M, Poeppel TD, Bockisch A, Rosenbaum-Krumme S. Imaging of Prostate-Specific Membrane Antigen Expression in Metastatic Differentiated Thyroid Cancer Using 68GaHBED-CC-PSMA PET/CT. Clin Nucl Med. 2017; 42:20 25. https://doi.org/10.1097/RLU.0000000000001454.

21. Wang W, Tavora F, Sharma R, Eisenberger M, Netto GJ. PSMA expression in Schwannoma: a potential clinical mimicker of metastatic prostate carcinoma. Urol Oncol. 2009; 27:525-8. https://doi.org/10.1016/j. urolonc.2008.04.007.

22. Zeng C, Ke ZF, Yang Z, Wang Z, Yang SC, Luo CQ, Wang LT. Prostate-specific membrane antigen: a new potential prognostic marker of osteosarcoma. Med Oncol. 2012; 29:2234-9. https://doi.org/10.1007/s12032-011-0089-4.

23. Heitkotter B, Trautmann M, Grunewald I, Bogemann M, Rahbar K, Gevensleben H, Wardelmann E, Hartmann W, Steinestel K, Huss S. Expression of PSMA in tumor neovasculature of high grade sarcomas including synovial sarcoma, rhabdomyosarcoma, undifferentiated sarcoma and MPNST. Oncotarget. 2017; 8:4268-76. https://doi. org/10.18632/oncotarget.13994.

24. Kanthan GL, Drummond J, Schembri GP, Izard MA, Hsiao E. Follicular Thyroid Adenoma Showing Avid Uptake on 68Ga PSMA-HBED-CC PET/CT. Clin Nucl Med. 2016; 41:331-2. https://doi.org/10.1097/ RLU.0000000000001084

25. Bychkov A, Vutrapongwatana U, Tepmongkol S, Keelawat S. PSMA expression by microvasculature of thyroid tumors - Potential implications for PSMA theranostics. Sci Rep. 2017; 7:5202. https://doi.org/10.1038/s41598-017-05481-z.

26. Rahbar K, Weckesser M, Huss S, Semjonow A, Breyholz HJ, Schrader AJ, Schafers M, Bogemann M.
Correlation of Intraprostatic Tumor Extent with (6)(8) Ga-PSMA Distribution in Patients with Prostate Cancer. J Nucl Med. 2016; 57:563-7. https://doi.org/10.2967/ jnumed.115.169243.

27. Ahmadzadehfar H, Eppard E, Kürpig S, Fimmers R, Yordanova A, Schlenkhoff CD, Gärtner F, Rogenhofer $\mathrm{S}$, Essler M. Therapeutic response and side effects of repeated radioligand therapy with $177 \mathrm{Lu}-\mathrm{PSMA}-$ DKFZ-617 of castrate-resistant metastatic prostate cancer. Oncotarget. 2016; 7:12477-88. https://doi.org/10.18632/ oncotarget. 7245 .

28. Milowsky MI, Nanus DM, Kostakoglu L, Sheehan CE, Vallabhajosula S, Goldsmith SJ, Ross JS, Bander NH. Vascular targeted therapy with anti-prostate-specific membrane antigen monoclonal antibody J591 in advanced solid tumors. J Clin Oncol. 2007; 25:540-7. https://doi. org/10.1200/JCO.2006.07.8097.

29. Von Hoff DD, Mita MM, Ramanathan RK, Weiss GJ, Mita AC, LoRusso PM, Burris HA 3rd, Hart LL, Low SC, Parsons DM, Zale SE, Summa JM, Youssoufian H, Sachdev JC. Phase I Study of PSMA-Targeted Docetaxel-Containing Nanoparticle BIND-014 in Patients with Advanced Solid Tumors. Clin Cancer Res. 2016; 22:3157-63. https://doi. org/10.1158/1078-0432.CCR-15-2548.

30. Fina L, Molgaard HV, Robertson D, Bradley NJ, Monaghan P, Delia D, Sutherland DR, Baker MA, Greaves MF. Expression of the CD34 gene in vascular endothelial cells. Blood. 1990; 75:2417-26.

31. Schlingemann RO, Rietveld FJ, de Waal RM, Bradley NJ, Skene AI, Davies AJ, Greaves MF, Denekamp J, Ruiter DJ. Leukocyte antigen CD34 is expressed by a subset of cultured endothelial cells and on endothelial abluminal microprocesses in the tumor stroma. Lab Invest. 1990; 62:690-6.

32. Siemerink MJ, Klaassen I, Vogels IM, Griffioen AW, Van Noorden CJ, Schlingemann RO. CD34 marks angiogenic tip cells in human vascular endothelial cell cultures. Angiogenesis. 2012; 15:151-63. https://doi.org/10.1007/ s10456-011-9251-z.

33. Schildhaus HU, Riegel R, Hartmann W, Steiner S, Wardelmann E, Merkelbach-Bruse S, Tanaka S, Sonobe H, Schule R, Buettner R, Kirfel J. Lysine-specific demethylase 1 is highly expressed in solitary fibrous tumors, synovial sarcomas, rhabdomyosarcomas, desmoplastic small round cell tumors, and malignant peripheral nerve sheath tumors. Hum Pathol. 2011; 42:1667-75. https://doi.org/10.1016/j. humpath.2010.12.025. 